\title{
EPIDEMIOLOGICAL DATA AND CANCER RISK ASSESSMENT: CESSATION LAG AND LINGERING EFFECT CONCEPTS
}

\author{
Vladimír Bencko', Chao Chen² \\ ${ }^{1}$ Institute of Hygiene and Epidemiology, First Faculty of Medicine, Charles University in Prague, Prague, Czech Republic \\ 2US Environmental Protection Agency, Washington, D.C., USA
}

\begin{abstract}
SUMMARY
Cessation lag and lingering effect are two important aspects of risk assessment, and have potential applications to dose-response analysis in risk assessment. In addition to providing insight into biological mode of action, the concept of cessation lag is useful for economic benefit analysis. Concept of effect lingering can be used to analyze epidemiological data by uncovering the hidden biological implications related to disease endpoints, thereby advancing current efforts to characterize and reduce risk assessment uncertainties.

Multicentre study design is proposed as a way to increase study size and to mitigate criticism of meta-analysis of independent studies. Individual studies from a multicentre study can be either pooled using original data, or combined by meta-analysis of summarized results. A multicentre study of large cohort or case-control study also offers an exciting opportunity to study the contribution of epigenetic events that may be associated with life-style and environmental risk factors for human health. Methods for optimizing exposure assessment and reducing exposure misclassification represent important but difficult components in epidemiological studies. Biomarkers present a potentially useful approach for improving exposure estimates.
\end{abstract}

Key words: epidemiological data, carcinogenicity, multicentre studies, lingering effect, cessation lag, risk assessment

Address for correspondence: V. Bencko, Charles University in Prague, 1st Faculty of Medicine, Institute of Hygiene and Epidemiology, Studničkova 7, Praha 2, Czech Republic. E-mail: vladimir.bencko@lf1.cuni.cz

\section{INTRODUCTION}

The field of epidemiology has reached a crucial point with challenges and opportunities. On one hand, it seems that most of the major occupational carcinogens have already been identified. Many chemicals classified as carcinogens by the International Agency for Research on Cancer (IARC) were first evaluated in the workplace. In the last decades, occupational exposure to known human carcinogens has diminished in many countries as awareness of their hazards has increased. On the other hand, we are still confronted with a long list of substances for which epidemiological data are lacking or inconclusive. The estimated number of chemicals in commerce ranges from tens of thousands to over 140,000; for most of them, relevant toxicological information is needed to set regulatory standards (1-3).

We are now at an important crossroad; advances in the interrelated disciplines on which health risk assessment depends hold promise for comprehensive understanding of the influence of environmental stressors on human health. The last decade has been marked with major developments in the field of cancer risk assessment. There have been remarkable advances in the broad area of cancer epidemiology. This includes research not only on human exposures to major cancer risk factors in environmental and occupational settings, but also on lifestyle and nutrition related risks. Traditional approaches and study design in cancer epidemiology have not been successful in identifying and evaluating these potential risk and/or protective factors.
Two main reasons for this failure are often due to insufficient study size and inadequate exposure assessment. In this paper, we discuss issues and approaches relevant to these two challenges, and the opportunity of using emerging genomics information in epidemiology studies.

\section{Increasing Study Size}

An important characteristic of research in the last decade is the increasing number of collaborative studies involving various countries, and as a consequence, sample size is greatly increased. By increasing the sample size, the power of a study to identify significant associations between exposures and a disease endpoint is enhanced. For example, considering that lifetime prevalence of occupation-related exposures in the population is low (typically below 5 or $10 \%$ ), and the associated risk can be small as well (e.g. relative risk of 2). The study sample size for a community based study must be large in order to identify statistically significant associations. This is even more crucial if the exposure or disease outcome is misclassified in a study population. A sample size of at least 1000 cases and controls has been recommended for a community-based case-control study on cancer. However, it is often the case that one centre or one country cannot provide such a number of cases within a reasonable amount of time. Therefore, multicentre studies are an obvious solution. Besides increased power, multicentre studies can provide additional advantages that include greater exposure variation in the study population which 
is useful for dose-response analysis, and an opportunity to study differences in exposure and disease patterns between countries.

Multicentre studies can be created in two different ways. Collaboration between centres can be initiated after completion of each individual study (retrospectively planned multicentre studies), or before the studies have actually started (prospectively planned multicentre studies). Meta- and pooled analyses are examples of retrospectively planned multi-centre studies, and have been performed in many areas of epidemiology. Prospectively planned multicentre studies have only relatively recently become possible since international organizations and institutions such as the European Commission started to offer funding for these costly operations. The advantage these studies offer is an identical protocol for data collection at each centre involved, while avoiding loss of information at the stage of data pooling. From the Central and East European Countries (CEEC) perspective, an important example of this approach is the CEEC Multicenter Lung Cancer Study that was organized by IARC/WHO/Lyon, France, about 15 years ago, and was supported by EC Inco Copernicus FP4. It represented collaboration of centres in the Czech Republic, Hungary, Poland, Romania, Russian Federation, and Slovakia (4-7). Later, the same organizational principle was applied in the Kidney Cancer Study supported by NCI/Bethesda, USA $(8,9)$.

A great advantage of multicentre studies is that the potentially serious problems associated with the use of meta-analysis in observational studies can now be substantially mitigated. The merit of applying meta-analysis to observational studies has been questioned and controversial $(10,11)$ because errors and biases can be easily introduced when studies with different designs, methods and population characteristics are combined. It would not be a problem for pooled or meta-analysis if the study design is comparable, and method for data collection is coordinated prior to the initiation of the study among different centres. There are many factors that make meta-analysis of individual studies less appropriate. Consider, for example, meta-analysis of odd ratios or relative risk estimates that require auxiliary information such as age, sex, smoking status, and sample size. If all auxiliary variables are homogenous across studies, it would be appropriate to conduct meta-analyses of these studies $(12,13)$. In reality, these conditions have never been satisfied. Therefore, the multicentre study design offers an attractive alternative to the traditional single-centre epidemiological study.

\section{Improving Exposure Assessment}

Optimizing methods for exposure assessment and reducing exposure misclassification represent the most difficult components of epidemiological studies. A perfect exposure assessment for long latency diseases such as cancer would consist of quantitative measurements of internal dose, over the whole exposure period, for each subject in the study. This is a utopia unlikely to be attained in the near future. The availability of group-based external exposure measurements at a few points in time is already a luxurious proposition for epidemiologists. More often one has to fall back on subjective methods of exposure assessment. The possibilities for exposure assessment largely depend on the design of the epidemiological study, with community-based and industry-based studies imposing their own specific limitations. In community-based case-control studies, occupational chemi- cal exposures of interest often cannot be measured directly, and have to be estimated retrospectively. As a consequence, exposure estimates are often based directly on the job information provided by the study subjects (or proxies), or inferred from the job information through job exposure matrices, or by expert assessment case-by-case. The subjectivity and the limited use of data-driven quantitative estimates of exposure used in case-control studies are considered important shortcomings that can lead to substantial exposure misclassification. How to improve retrospective exposure assessment methods has been of frequent debate. The difficulty in evaluating the validity of all retrospective exposure assessment methods in the absence of a gold standard is considered a major shortcoming.

Reliability studies indicate that a considerable amount of misclassification can occur in all retrospective methods (14-16). There is, however, an urgent need to quantify levels of misclassification expected from each method in order to anticipate the attenuation of the resultant odds ratio (OR) estimates. A major area of improvement needed is the departure from crude exposure indicators such as never/ever exposed towards a more quantitative exposure assessment covering the entire exposure period. Quantitative exposure data that enable the investigation of the exposure-response relationship is an important criterion for causation. The availability of quantitative exposure data also facilitates valid comparison of risk and exposure-response relations among studies, countries and industries, and provides a solid base for risk assessment and standard setting.

A potentially useful approach for improving exposure estimate is to use biomarkers of exposures. Biomarkers of exposure have been used in health risk assessment for a long time but its potential use in risk assessment has not been fully exploited. The utility of biomarkers is greatly enhanced when body burden resulted from multiple source exposures, or when the source of exposure is difficult to identify (e.g. exposure to second hand smoke). There are situations where biomarkers of exposures could provide valuable information in addition to traditional measurement of exposures in epidemiological studies. For instance, when data obtained from studies of a community exposed to elevated arsenic contamination in drinking water is used for risk assessment, a contentious issue is: what is the total arsenic intake for a person? Assessment of exposure is often based on the measured concentrations in the drinking water, estimations of the amount of water consumed and used for cooking, and an estimate of dietary intake. Under these uncertainties, biological monitoring offers a useful solution as biomarkers of exposure providing data on the absorbed dose for each individual. Biomarkers may include arsenic in urine, blood, hair, and nails. However, suitability of the various biomarkers to serve as indicators of, in this case, a chronic exposure to inorganic arsenic and the various factors needs to be carefully evaluated $(17,18)$ and could also serve to make results comparable for a given endpoint with other community-based studies.

\section{Epigenomics - New Challenges and Opportunities}

While the field of cancer genetics has experienced a great deal of attention among cancer researchers in the last few decades (2, 3 ), appreciation of cancer epigenetics is more recent. The study of the role of epigenetic changes induced by environmental, dietary and lifestyle factors is rapidly emerging but still in its infancy. 
Little is known about the precise contribution of epigenetic mechanisms to human health effects induced by adverse environmental and dietary stimuli. While there is accumulating evidence showing that aberrant DNA methylation may result from adverse exposures to epimutagens, there is a paucity of evidence regarding the effects of stimuli causing heritable changes in epigenetic information stored in histones. This is because epigenomics is a new and largely unexplored field. Although it seems inevitable that perturbations in histone modifications are induced by dietary and environmental factors that contribute to the development of human disease such as cancers, a rigorous proof of such a relationship remains to be established (19).

Multicenter and large cohort or case-control studies offer some of the most exciting opportunities to study the contribution of epigenetic events induced by the diet and environment to human cancer. Such examples are the objectives of the European Prospective Investigation into Cancer and Nutrition: a large prospective cohort study designed to investigate the relationship between diet, lifestyle, and the incidence of cancer in 10 European countries (20); and a case-control study on lung and upper aerodigestive tract cancers in Central and Eastern Europe (21-23). These multicentre studies boast a large sample size of several thousand subjects and represent a unique opportunity to identify dietary/ lifestyle practices and environmental stimuli which may exert risk and/or benefit effects through epigenomic changes. Epigenetic alterations in comparison with genetic changes are reversible and are typically acquired in a gradual manner. These features offer an important potential opportunity for prevention strategies (19).

Risk Assessment, Cessation Lag and Lingering Effect

Risk assessment is an evolving science, and methods for conducting risk assessment are still in their infancy, although undergoing rapid development. Cessation lag and lingering effect are two important aspects of risk assessment, and have potential applications to dose-response analysis in risk assessment $(24,25)$. In addition to providing insight into biological mode of action, the concept of cessation lag is useful for economic benefit analysis. Concept of effect lingering can be used to analyze epidemiological data by uncovering the hidden biological implications related to disease endpoints, thereby advancing current efforts to characterize and reduce risk assessment uncertainties.

It is useful to introduce concepts of cessation lag, and lingering effect, and to analyze an epidemiological data set from the view of understanding these aspects. Environmental regulations aimed at reducing cancer risks usually have the effect of reducing exposure to a carcinogen at the time the regulation is implemented. The reduction of cancer risk may occur shortly after the reduced exposure or after a considerable period of time. Some questions of interest in risk assessment and economic analysis are: 1) How many cancer cases are avoided at age $t$ after cessation (or reduction) of exposure concentration? 2) How long does the effect of an exposure last after exposure has terminated (or been reduced)? Lingering effect is the term used to describe exposure effects that continue into the future. Lingering effect can be defined as remaining hazard after occurrence of an exposure. Therefore, lingering effect encompasses exposure effects that contribute to the subsequent disease hazard, at a particular age in life, after exposure. In other words, if the age-specific rate of a cancer depends only on current exposure, and all previous exposures are irrelevant, then there is no lingering effect.

It is important to distinguish among terms of latency period, cessation lag, and lingering effect. For cancers, latency period can be defined as the time from the first exposure to the occurrence (or observation) of a tumor, while cessation lag is defined as the time required for cancer risk to reach a steady level after cessation or reduction of exposure. According to the NCI Dictionary of Cancer Terms, the latency period is defined as: "The time that passes between being exposed to something that can cause disease (such as radiation or a virus) and having symptoms." In other words, latency period that represents time to a cancer diagnosis is a random variable with a probability distribution. However, there is rarely a study that investigates cancer latency period under such a rigorous conceptual principle. To avoid lengthy discussions here, readers who are interested in concepts of cessation lag and lingering effect should consult their original publications $(24,25)$. In hindsight, it seems rather surprising to find that most epidemiological analyses for risk assessment have focused on latency rather than on cessation lag or lingering effect. A good example of lingering effect is the radiation-associated health effects present in atomic bomb survivors in Japan. Fifty-five years later, researchers still observe the health effects due to the radiation exposure of the atomic bomb. Thyroid diseases, including tumors, are still found to present and positively related to the radiation levels among atomic bomb survivors. Researchers have learned a great deal of scientific knowledge from the lingering effects associated with the single atomic bomb explosion. Under this exposure scenario, the latency period is determined as the time to the first observed tumor of interest. In this case, lingering effect and latency can be easily evaluated from the data. The challenge is how to extract similarly useful information from other epidemiological data involving multiple exposures. The concept and methodologies for investigating these issues can be found in papers by Chen and Gibb (24) and Chen (25).

Controversies abound concerning the appropriate methods and useful data, great uncertainties involved in extrapolating beyond the range of available data, underlying biases and other limitations of observational data (26-29), and political and societal implications of these analyses (3). Sceptics have argued that risk assessment, at least as it is currently practiced, has not been a useful tool for addressing societal concerns about exposures to environmental and occupational hazards (30). Their primary concern is that increasingly intense debates concerning risk assessments may come to be used as an excuse for delay in the development of appropriate regulatory and other responses to environmental and occupational hazards. For example, it has taken the U.S. EPA more than 20 years to finalize its risk assessment for exposure to diesel exhaust particulates (31).

Identification and quantification of risks is clearly a useful tool for informed decision-making. Appropriately, this is also the underlying principle for the current U.S. EPA's default approach for cancer risk assessment, in the absence of adequate scientific information useful for quantitative risk estimation. This is not a new principle for epidemiologists. Risk assessments are inherently uncertain and should, as the National Academy of Sciences (32) suggested, be viewed as an iterative process in need of continual improvements through research targeted to fill the gaps in our evidence based knowledge. Tremendous advances in 
our understanding of basic epigenetic mechanisms and rapidly developing new powerful technologies, such as those for sensitive and quantitative detection of epigenetic changes as well as for genome-wide analysis (epigenomics), hold great promise that these issues may be addressed in the near future (19).

\section{CONCLUSIONS}

Epidemiological data play a crucial role in the desirable evidence-based cancer risk assessment and classification of human carcinogens. With the emerging genomic and molecular data and the adoption of the multicentre study concept, there is a real hope that the foreseeable future will bring long awaited answers to the problems we encounter today, such as the impact of aberrant genetic and epigenetic interaction with environment and diet. New biological information could lead to discovery of new biomarkers and development of novel strategies for health risk analysis useful for disease prevention, a major public health priority in the 21 st century.

\section{Acknowledgement}

The paper was written within activities supported by the project PRVOUK-P28/1FF/6 and the League Against Cancer, Prague.

\section{REFERENCES}

1. Stayner L, Boffetta P, Vainio H. Risk assessment of carcinogenic hazards. In: Schottenfeld D, Fraumeni JF Jr, editors. Cancer epidemiology and prevention. 3rd ed. Oxford: Oxford University Press; 2006. p. 65-9.

2. Bencko V. Environmental epidemiology, present chances and challenges for future. Cent Eur J Public Health. 2007 Nov;15 Suppl:S6-8.

3. Bencko V. Use of epidemiological data for cancer risk assessment: approaches, concepts and issues. Open Epidemiol J. 2011 Jan 19;4:94-8.

4. Gemignani F, Landi S, Szeszenia-Dabrowska N, Zaridze D, Lissowska J, Rudnai P, et al. Development of lung cancer before the age of 50: the role of xenobiotic metabolizing genes. Carcinogenesis. 2007 Jun;28(6):128793.

5. Hung RJ, Hashibe M, McKay J, Gaborieau V, Szeszenia-Dabrowska $\mathrm{N}$, Zaridze D, et al. Folate-related genes and the risk of tobacco-related cancers in Central Europe. Carcinogenesis. 2007 Jun;28(6):1334-40.

6. Brennan P, Hsu CC, Moullan N, Szeszenia-Dabrowska N, Lissowska J, Zaridze D, et al. Effect of cruciferous vegetables on lung cancer in patients stratified by genetic status: a mendelian randomisation approach. Lancet. 2005;366(9496):1558-60.

7. Brennan P, McKay J, Moore L, Zaridze D, Mukeria A, Szeszenia-Dabrowska N, et al. Obesity and cancer: Mendelian randomization approach utilizing the FTO genotype. Int J Epidemiol. 2009 Aug;38(4):971-5.

8. Moore LE, Brennan P, Karami S, Hung RJ, Hsu C, Boffetta P, et al. Glutathione S-transferase polymorphisms, cruciferous vegetable intake and cancer risk in the Central and Eastern European Kidney Cancer Study. Carcinogenesis. 2007 Sep;28(9):1960-4.

9. Karami S, Brennan P, Hung RJ, Boffetta P, Toro J, Wilson RT, et al. Vitamin D receptor polymorphisms and renal cancer risk in Central and Eastern Europe. J Toxicol Environ Health A. 2008;71(6):367-72.

10. Chalmers TC. Problems induced by meta-analyses. Stat Med. 1991 Jun;10(6):971-9; discussion 979-80.

11. Thompson SG, Pocock SJ. Can meta-analyses be trusted? Lancet. 1991 Nov 2;338(8775):1127-30.
12. Blettner M, Sauerbrei W, Schlehofer B, Scheuchenpflug T, Friedenreich C. Traditional reviews, meta-analyses and pooled analyses in epidemiology. Int J Epidemiol. 1999 Feb;28(1):1-9.

13. Stroup DF, Berlin JA, Morton SC, Olkin I, Williamson GD, Rennie D, et al., Meta-analysis Of Observational Studies in Epidemiology (MOOSE) Group. Meta-analysis of observational studies in epidemiology: a proposal for reporting. JAMA. 2000 Apr 19;283(15):2008-12.

14. Benke G, Sim M, Forbes A, Salzberg M. Retrospective assessment of occupational exposure to chemicals in community-based studies: validity and repeatability of industrial hygiene panel ratings. Int J Epidemiol. 1997 Jun;26(3):635-42.

15. 't Mannetje A, Fevotte J, Fletcher T, Brennan P, Legoza J, Szeremi M, et al. Assessing exposure misclassification by expert assessment in multicenter occupational studies. Epidemiology. 2003 Sep;14(5):585-92.

16. Blair A, Stewart P, Lubin JH, Forastiere F. Methodological issues regarding confounding and exposure misclassification in epidemiological studies of occupational exposures. Am J Ind Med. 2007 Mar;50(3):199-207.

17. Bencko V, Symon K. Health aspects of burning coal with a high arsenic content. I. Arsenic in hair, urine, and blood in children residing in a polluted area. Environ Res. 1977 Jun;13(3):378-85.

18. Uchino T, Roychowdhury $\mathrm{T}$, Ando M, Tokunaga $\mathrm{H}$. Intake of arsenic from water, food composites and excretion through urine, hair from a studied population in West Bengal, India. Food Chem Toxicol. 2006 Apr;44(4):455-61.

19. Herceg Z. Epigenetics and cancer: towards an evaluation of the impact of environmental and dietary factors. Mutagenesis. 2007 Mar;22(2):91-103.

20. Riboli E, Kaaks R. The EPIC Project: rationale and study design. Int J Epidemiol. 1997;26 Suppl 1:S6-14.

21. Hung RJ, Brennan P, Canzian F, Szeszenia-Dabrowska N, Zaridze D, Lissowska J, et al. Large-scale investigation of base excision repair genetic polymorphisms and lung cancer risk in a multicenter study. J Natl Cancer Inst. 2005 Apr 20;97(8):567-76.

22. Hung RJ, van der Hel O, Tavtigian SV, Brennan P, Boffetta P, Hashibe M. Perspectives on the molecular epidemiology of aerodigestive tract cancers. Mutat Res. 2005 Dec 30;592(1-2):102-18.

23. Scélo G, Constantinescu V, Csiki I, Zaridze D, Szeszenia-Dabrowska N, Rudnai P, et al. Occupational exposure to vinyl chloride, acrylonitrile and styrene and lung cancer risk (Europe). Cancer Causes Control. 2004 Jun;15(5):445-52.

24. Chen CW, Gibb H. Procedures for calculating cessation lag. Regul Toxicol Pharmacol. 2003 Oct;38(2):157-65.

25. Chen CW. Lingering effect: Epidemiological information useful for risk assessment. Regul Toxicol Pharmacol. 2008 Dec;52(3):242-7.

26. Bencko V. Risk assessment and human exposure to endocrine disrupters. In: Jedrychowski WA, Perera FP, Maugeri U, editors. Molecular epidemiology in preventive medicine. Luxemburg: International Center for Studies and Research in Biomedicine; 2003. p. 315-27.

27. Bencko V, Rames J, Van Den Berg M, Plesko I, Trnovec T. Human exposure to polyhalogenated hydrocarbons and incidence of selected malignancies. In: Donnelly KC, Cizmas LH, editors. Environmental health in Central and Eastern Europe. Dordrecht: Springer; 2006. p. 31-7.

28. Sasco AJ. Cancer and globalization. Biomed Pharmacother. 2008 Feb;62(2):110-21

29. Bencko V, Rames J, Ondrusova M, Plesko I, Jurickova L, Trnovec T. Human exposure to polyhalogenated hydrocarbons and incidence of selected malignancies - central European experience. Neoplasma. 2009;56(4):3537 .

30. Silbergeld EK. Risk assessment: the perspective and experience of U.S. environmentalists. Environ Health Perspect. 1993 Jun;101(2):100-4.

31. Stayner L. Protecting public health in the face of uncertain risks: the example of diesel exhaust. Am J Public Health. 1999 Jul;89(7):991-3.

32. National Research Council. Arsenic in drinking water. Washington, DC: National Academic Press; 1999. 